\title{
Self-Organization and Annealed Disorder in a Fracturing Process
}

\author{
Caldarelli, Guido; Di Tolla, Francesco; Petri, Alberto
}

Published in:

Physical Review Letters

Link to article, DOI:

10.1103/PhysRevLett.77.2503

Publication date:

1996

Document Version

Publisher's PDF, also known as Version of record

Link back to DTU Orbit

Citation (APA):

Caldarelli, G., Di Tolla, F., \& Petri, A. (1996). Self-Organization and Annealed Disorder in a Fracturing Process. Physical Review Letters, 77(12), 2503-2506. https://doi.org/10.1103/PhysRevLett.77.2503

\section{General rights}

Copyright and moral rights for the publications made accessible in the public portal are retained by the authors and/or other copyright owners and it is a condition of accessing publications that users recognise and abide by the legal requirements associated with these rights.

- Users may download and print one copy of any publication from the public portal for the purpose of private study or research.

- You may not further distribute the material or use it for any profit-making activity or commercial gain

- You may freely distribute the URL identifying the publication in the public portal

If you believe that this document breaches copyright please contact us providing details, and we will remove access to the work immediately and investigate your claim 


\title{
Self-Organization and Annealed Disorder in a Fracturing Process
}

\author{
Guido Caldarelli \\ I.N.F.M., Istituto Nazionale di Fisica della Materia, Sezione di Trieste, Scuola Internazionale Superiore di Studi \\ Avanzati/International School for Advanced Study, v. Beirut 2-4, I-34013 Grignano di Trieste (TS), Italy \\ Francesco D. Di Tolla \\ Scuola Internazionale Superiore di Studi Avanzati/International School for Advanced Study, v. Beirut 2-4, \\ I-34013 Grignano di Trieste (TS), Italy \\ and Center for Atomic-scale Materials Physics, Technical University of Denmark, DK-2800 Lyngby, Denmark
}

\author{
Alberto Petri \\ Consiglio Nazionale delle Ricerche, Istituto di Acustica “O.M. Corbino,” v. Cassia 1216, I-00189 Roma, Italy \\ and I.N.F.N., Istituto Nazionale di Fisica Nucleare, Sez. di Perugia, Italy
}

(Received 21 February 1996)

\begin{abstract}
We show that a vectorial model for inhomogeneous elastic media self-organizes under external stress. An onset of crack avalanches of every duration and length scale compatible with the lattice size is observed. The behavior is driven by the introduction of annealed disorder, i.e., by lowering the breaking threshold in the neighborhood of a bond broken by the stress, with a process similar to self-organized criticality. A further comparison with experimental results of acoustic emission (AE), shows that the stability of the elastic potential energy of the system in the AE regime is a sufficient condition for reproducing the algebraic distribution of the energy released during cracks formation. [S0031-9007(96)01196-9]
\end{abstract}

PACS numbers: 64.60.Lx, 05.40.+j, 46.30.Nz, 62.20.Mk

Since the concept of self-organized criticality (SOC) was introduced by Bak, Tang, and Wiesenfeld [1], an increasing number of physical situations showing this behavior has been studied. In fact, SOC is observed in a variety of phenomena ranging from river evolution [2] and interface growth [3-5] to biological evolution [6]. In analogy with usual critical systems, the behavior of SOC systems is characterized by the onset of long range correlations.

In common statistical systems, criticality is obtained only after finely tuning some external parameter, e.g., temperature. On the contrary, in SOC systems there is no evidence of such a parameter, and instabilities occur when the value of the relevant field exceeds locally some threshold value (rigidity). Thus according to a diffusive rule, the field perturbation spreads over the neighbors, which in turn can be set into a metastable state (above their local threshold) and can relax over further neighbors, and so on. This can trigger avalanches of any size and duration producing the characteristic power law frequency distributions, due to the lacking of characteristic scales in the process, i.e., self-similarity. Since there is no parameter to be adjusted in order to reach criticality, one says that the system self-organizes into a critical state.

Statistical self-similarity has been observed for a long time in seismic events [7], as well as in energy release by material fracturing [8] and many other different systems subjected to stress [9]. It is therefore very tempting to investigate the possibility that the same critical dynamics holds from the large scale of earthquakes down to the microscopic scale of the rheological properties of materials. In that case, as in usual critical phenomena, the detailed aspects of different structures would be irrelevant with respect to the critical properties.

In this work we try to specify the conditions under which self-organization takes place in stressed materials and to what extent it can explain the intriguing experimental properties of the related acoustic emission (AE). In fact, the current understanding of dissipation in solids is largely phenomenological. A well known feature that determines the rupture process is the presence of disorder in the material. These inhomogeneities strongly influence the mechanical behavior of the material and are responsible for the experimental patterns of $\mathrm{AE}$, proving that energy dissipation occurs in avalanches, whose size ranges from the scale of microscopic perturbations to the large scale of the system size. Our purpose is to show that the onset of long range correlation in $\mathrm{AE}$ can be related to microscopical processes similar to those of simple SOC automata models. Specifically, a necessary condition for the $\mathrm{AE}$ power law behavior turns out to be the presence of annealed disorder in the system.

To model the medium we use a version of the Born model (BM) [10] that is a generalization of Hookean networks of springs $[11,12]$. Fractal and topological properties of BM have been investigated in the past $[13,14]$. We start by considering a triangular lattice of sites in which each pair of sites $i, j$ is connected by an elastic (brittle) spring to which is assigned an elongation threshold $R_{i, j}$. The elastic potential energy of the whole system is the sum 
over all the springs present in the lattice

$$
V=\frac{1}{2} \sum_{i, j} V_{i j}
$$

where the sum is over nearest neighbor sites, and $V_{i j}$ is the potential energy of the individual spring.

$$
V_{i j}=(\alpha-\beta)\left[\left(\vec{u}_{i}-\vec{u}_{j}\right) \cdot \vec{r}_{i j}\right]^{2}+\beta\left[\vec{u}_{i}-\vec{u}_{j}\right]^{2} .
$$

Here $\vec{u}_{i}$ is the displacement vector of site $i, \vec{r}_{i j}$ is the unit vector between the rest positions of sites $i, j$, and $\alpha$ and $\beta$ are force constants. We use a two dimensional lattice, with periodic boundary conditions in one direction and an applied tensile stress along the other. Then we relax the system to the global energy minimum keeping the boundaries fixed. Extensive numerical simulations are realized in the $\beta=0$ case, and some results for the $\beta \neq 0$ case are reported. We recover qualitatively the experimental $\mathrm{AE}$ behavior only when short range correlation between breaking thresholds, i.e., annealed disorder, is considered. The annealing acts on the neighbors of the broken springs lowering the rupture threshold of the springs nearest neighbors. This means that one rupture weakens the set of bonds around simulating corrosion behavior or damage spreading.

The breaking process is inspired to the rules of the simplest automata models prototypes of SOC. A flowchart schematizing the dynamics steps is in Fig. 1. Steps from 2 to 4 are performed again over the springs that had their threshold updated, in order to break them if their energy is found to be above the new threshold. If this is the case, more springs are broken and a new toppling occurs. One says than an "avalanche" is observed if one or more springs are broken in the system between two consecutive stretches. Hence we assign to each avalanche a size, an energy, and a duration. The size is taken as the total number of broken springs between two consecutive elongations. The corresponding energy is computed summing up the energy carried by these springs before their breakdown. The duration is determined as the number of consecutive relaxations (number of loops over steps 2-4) which the systems undergo before being further stretched (step 1). The whole sequence of previous steps is repeated until the sample is totally broken. According with the above rules, the energy of the system is stored in the springs and released with their rupture. To model the fracturing process, we start the simulations with a defectfree sample. We assign to each spring a failure threshold, uniformly distributed between 0 and a maximum value related to the total stress amount. In this work the behavior of the system is investigated in the limit of a vanishing stress rate for each step ( $0.5 \%$ of the total stress). In this way the dynamics of the lattice becomes very similar to the one investigated in Ref. [12].

In original sandpile models of SOC [1], sand grains are added on the top of a sandpile, causing instability. Every now and then a sand avalanche starts, and restores

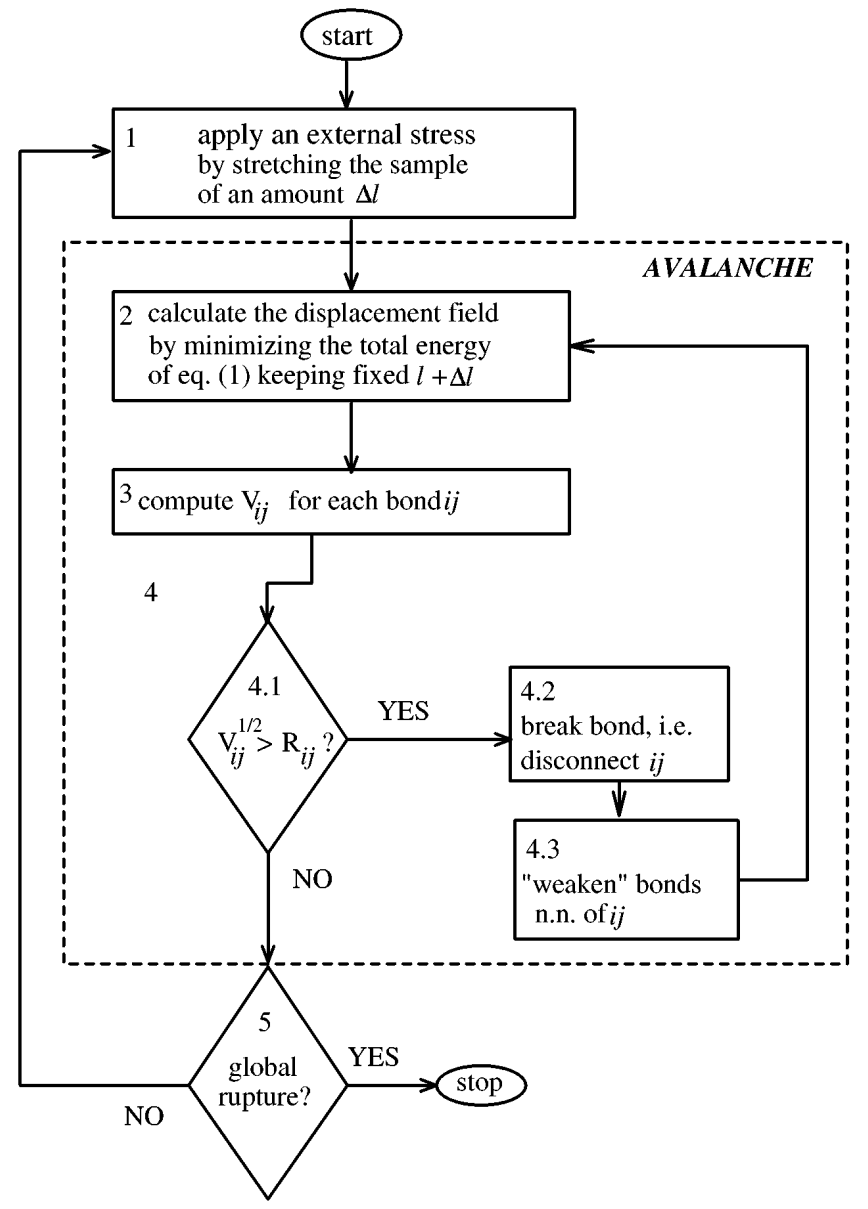

FIG. 1. Flowchart of the algorithm used in the simulations. The loop over steps 2 to 4 is repeated as long as new bonds are broken, and is what we define as an avalanche. The simulation ends when the sample is broken in two pieces.

equilibrium. As a consequence, the system is kept at criticality: The average slope or height does not change, but a small perturbation (added grain) may generate events of any extension in size and time (avalanches). The system stores locally (rigidity) potential energy of the incoming flux of sand and loses it releasing sand from the boundary. Similarly, in the case of elasticity, the applied stress strains the system, and more and more energy is stored in the springs. This energy is then released through the onset of cracks and in $\mathrm{AE}$. The equilibrium is restored through the relaxation.

We compute crack-avalanche statistics by recording the number of broken springs (the size of an avalanche), its duration, and its energy, i.e., the energy lost by the broken springs. We try to interpret this energy as the one released via $\mathrm{AE}$. In real experiments this is measured outside the sample and it is found to display similar power law distributions in many different materials and experimental situations $[8,9]$.

We find that when only quenched disorder is considered, no avalanche behavior is observed. This can be understood by noting that in the limit $\Delta l \rightarrow 0$, only one spring at time breaks, the weakest. This continues up to 
the breakdown of the system. This situation is analogous to that of the model for hydraulic fracturing investigated in [15]. In fact in that model no power law is found in the avalanche statistics. In this situation the system resembles the Duxbury-Leath [16] scenario. In fact when stress is applied, the failure instability initiates in an especially weak part of the material and the network stabilizes with a flaw population generated by the failure process. If the external stress increases by successive composition of infinitesimal amounts, one reaches (breaking one spring at a time) the critical stress for which the system undergoes complete failure.

Instead when annealed disorder is introduced, the situation changes drastically. The frequency distribution of crack sizes turns into a power law, reflecting the self-organization of $R_{i j}$ around the local stress values. Figure 2 shows the integrated distribution $P(N>n)$ of the number $N$ of broken springs to be greater than $n$, for lattices of different size. These data are fitted with a law $P(N>n)=A n^{-\tau+1}+B$ with an exponent $\tau=$ $2.00 \pm 0.05$. The annealed situation turns out to be close to the one described in Ref. [17], in which a model for the fracture of fibrous material is studied. In that model, a critical behavior is found when the influence of thermal fluctuations is comparable with the disorder due to the interaction among fibers.

Our interpretation of this different behavior with respect to the randomness distribution is that the breakdown threshold and the annealed disorder, respectively, play the role of the local rigidity and of the short-range couplings that, as pointed out in Ref. [18], are essential ingredients of SOC behavior. In fact in our case the breakdown threshold acts as the local rigidity (allowing local accumulation of stress), while the annealed disorder mimics the toppling

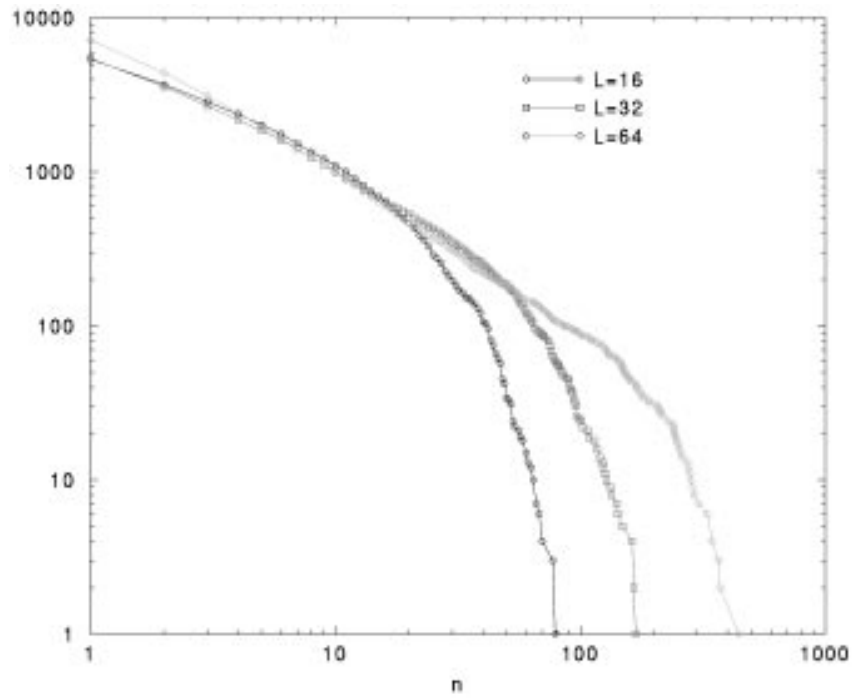

FIG. 2. Cumulated frequency distribution of the number of broken springs during an avalanche for different lattice sizes: 200 simulations for a $16 \times 16$ sites lattice; 100 simulations for a $32 \times 32$ sites lattice; 100 simulations for a $64 \times 64$ sites lattice. process in sandpiles (short range distribution of the stress), making the bonds closer to the breakdown value. If one of these ingredients is absent, systems behave in a diffusive way relaxing energies at the boundaries. More specifically, it is the presence of annealed disorder that allows the system to visit different metastable states, in which it stops because of the presence of rigidity. Otherwise, a situation similar to the one in Refs. $[12,15,16]$ would take place, where dynamics is ruled by extreme values in the probability distribution of breaking thresholds.

One can assume to have AE proportional to the energy of the springs broken during the crack evolution. By measuring the behavior of this quantity we do not obtain the power law behaviors experimentally observed [8]. In fact, one has to note that our finite system accumulates more and more energy in the springs not yet broken. This implies that AE is a function of time in this limit. Instead, in our model, breaking processes involving a given number of springs will contribute in the same way to the avalanche size statistics, independently from the different moment of the system evolution at which they happen. On the other hand, they will have a different weight in the statistics of the released energy, due to the fact that the variation of energy they induce is relatively large with respect to the total energy of our sample. Hence with the particular rules chosen for the local correlation, we expect that the system self-organizes (i.e., the $R_{i, j}$ 's arrange their values around the local stress) in order to maintain a power law only in the number of broken bonds. The energy drift gives rise to a correction term for the $\mathrm{AE}$ probability distribution, and it is reasonable that a power law can be recovered in the AE statistics by normalizing the energy of each avalanche to the actual total energy of the lattice at that time. The behavior of cumulated and normalized occurrence frequency, $P\left(E^{\prime}>e\right)$ versus $e$, is shown in Fig. 3. $e$ is the normalized energy defined as

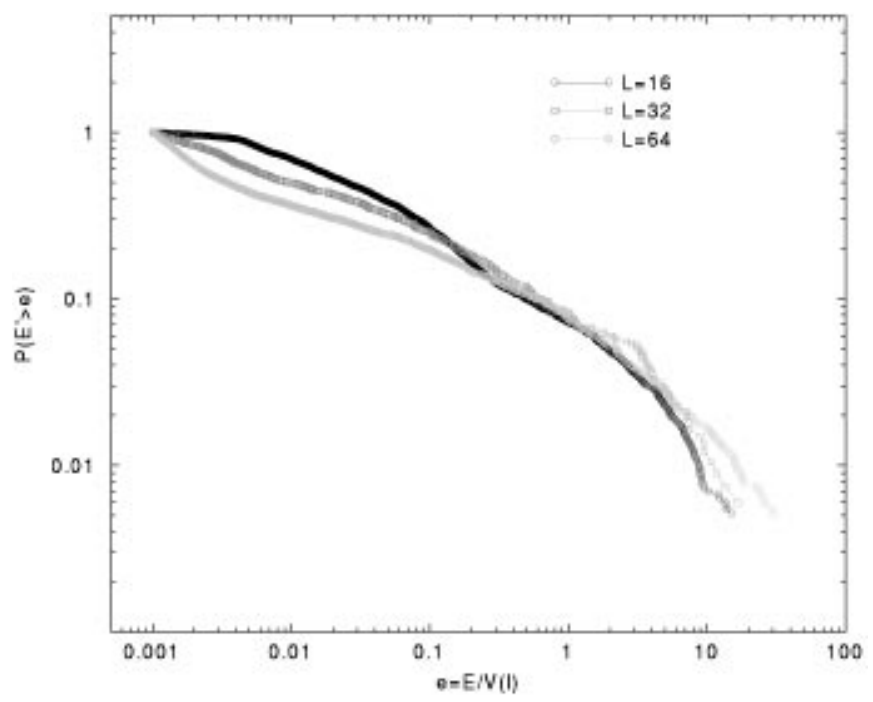

FIG. 3. Cumulated frequency distribution of the normalized energy lost during avalanches for the simulations of Fig. 2. 
$E / V(l), V(l)$ being the total potential energy defined in Eq. (1) at lattice elongation $l$, and $E$ being the sum of the $V_{i, j}$ for each bond $i, j$ broken. It is seen that, according to the above reasoning, the three curves relative to different lattice sizes collapse to a similar distribution. The fitting function $P\left(E^{\prime}>e\right)=A e^{-\gamma+1}+B$ gives $\gamma \approx 1.2-1.3$. Assuming that $e \sim n^{\rho}$, where $n$ stands for the number of broken bonds, a simple scaling relation yields $\rho=$ $(1-\tau) /(1-\gamma)$. With the previous values this brings to $E \approx V(l) n^{4}$ for the energy released. Then a power law distribution can be observed when the potential energy stored in the system does not vary too much during the AE process. This seems reasonable for most real systems, where AE starts when approaching the plastic regime.

Finally, we report the statistics of duration $t$ of crack avalanches, Fig. 4. A critical behavior $P(T>t) \sim t^{-\delta+1}$ is again observed, with an exponent $\delta=\tau=2.00 \pm 0.05$. We have performed simulations also for the cases $\beta=0.5$ and $\beta=1$. In our preliminary results we find the same exponents for size and duration of avalanches, i.e., $\tau=\delta=2$, whereas $\mathrm{AE}$ seems to be characterized by a little smaller exponent $\gamma \approx 1.1$.

In summary, we have introduced a generalized version of the Born model capable of describing a real physical situation for the AE [8] in which SOC takes part. Our principal results are as follows.

(a) Scale invariance arises when correlation (annealing) between the breaking of one bond and the bond's nearest neighbors is considered. The resulting competition with quenched disorder is closely related to SOC in the automata model, in which short range coupling and rigidity are necessary ingredients to set the system at some point of marginal stability. This is responsible for the critical behavior, as opposed to the extreme value statistics, typical of quenched disordered systems.

(b) Because of the particular chosen rules, critical behavior is displayed in the avalanche size statistics,

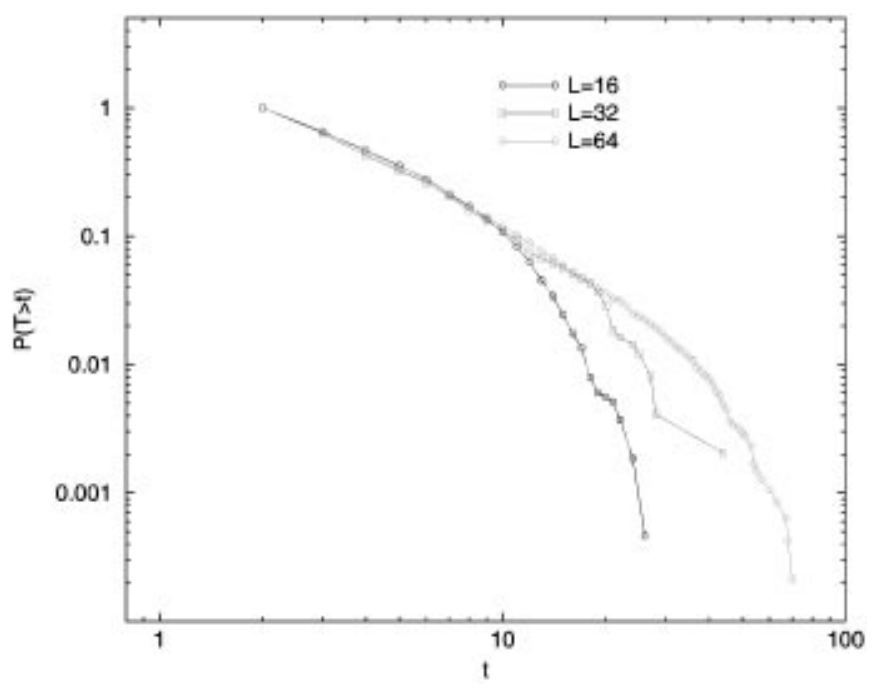

FIG. 4. Frequency distribution for the duration time of the avalanches for the simulations of Fig. 2. and not in AE. However, a self-similar probability distribution for this latter quantity is recovered when the ratio of the released energy to the total lattice energy (which sensibly increases with time in our model) is considered. This shows that a critical behavior of AE is compatible with systems where the elastic energy does not increase too much while straining. This is the situation of most real systems undergoing external macroscopic strains in the vicinity of the plastic regime.

(c) The above point allows us to relate these peculiar scaling processes to "slowly" developing fractures.

We remark that a more complete understanding of these processes is relevant not only for a more complete knowledge of the behavior of large statistical systems, but also from a technological point of view.

It is a pleasure to thank H. Herrmann, A. Maritan, S. Roux, A. Vespignani, and S. Zapperi for valuable discussions and suggestions.

[1] P. Bak, C. Tang, and K. Wiesenfeld, Phys. Rev. Lett. 59, 381 (1987); Phys. Rev. A 38, 364 (1988).

[2] A. Rinaldo, I. Rodriguez-Iturbe, R. Rigon, E. IjjaszVasquez, and R. L. Bras, Phys. Rev. Lett. 70, 822 (1993).

[3] K. Sneppen, Phys. Rev. Lett. 69, 3539 (1992); 71, 101 (1993).

[4] L.-H. Tang and H. Leschhorn, Phys. Rev. A 45, R8309 (1992).

[5] S. V. Buldyrev, A. L. Barabási, F. Caserta, S. Havlin, H. E. Stanley, and T. Vicsek, Phys. Rev. A 45, R8313 (1992).

[6] P. Bak and K. Sneppen, Phys. Rev. Lett. 71, 4083 (1993).

[7] J. M. Carlson and J.S. Langer, Phys. Rev. Lett. 62, 2632 (1989).

[8] A. Petri, G. Paparo, A. Vespignani, A. Alippi, and M. Costantini, Phys. Rev. Lett. 73, 3423 (1994); P. Diodati, F. Marchesoni, and S. Piazza, Phys. Rev. Lett. 67, 2239 (1991).

[9] Eric M. Kramer and Alexander E. Lobkovsky, Babbage Report No. condmat/9510090; Paul A. Houle and James P. Sethna, Phys. Rev. E 54, 278 (1996).

[10] E. Louis and F. Guinea, Europhys. Lett. 3, 871 (1987).

[11] L. de Arcangelis, A. Hansen, S. Roux, and H. J. Herrmann, Phys. Rev. B 40, 877 (1989).

[12] A. Hansen, S. Roux, and H. J. Herrmann, J. Phys. (Paris) 50, 733 (1989).

[13] H. Yan, G. Li, and L. M. Sander, Europhys. Lett. 10, 7 (1989).

[14] G. Caldarelli, C. Castellano, and A. Vespignani, Phys. Rev. E 49, 2673 (1994).

[15] F. Tzschichholz and H.J. Herrmann, Phys. Rev. E 51, 1961 (1995).

[16] P. M. Duxbury and P. L. Leath, Phys. Rev. Lett. 72, 2805 (1994).

[17] A. T. Bernardes and J. G. Moreira, Phys. Rev. B 49, 15035 (1994); (to be published).

[18] R. Cafiero, V. Loreto, L. Pietronero, A. Vespignani, and S. Zapperi, Europhys. Lett. 29, 111 (1995). 